\title{
Análise da assistência farmacêutica no planejamento: participação dos profissionais e a qualificação da gestão
}

\author{
Analysis of pharmaceutical assistance in planning: \\ participation of professionals and qualification of management
}

\author{
Letisa Comparin Dalla Nora ${ }^{1}$ (1), Karen Sarmento Costa $^{2}$ (1), \\ Suetônio Queiroz Araújoº (10, Noemia Urruth Leão Tavares ${ }^{4}$ (1)
}

\begin{abstract}
Resumo
Introdução: O desenvolvimento da assistência farmacêutica, assim como de todas as áreas de atuação do SUS, é de responsabilidade das três instâncias gestoras (municipal, estadual e federal) e, nesse contexto, é fundamental a participação dos atores responsáveis pela sua gestão no planejamento da previsão de recursos orçamentários próprios para alocação nessa área, conforme sua capacidade e prioridade, levando-se em conta a escassez de recursos e as necessidades verificadas. Objetivo: Descrever a participação dos profissionais responsáveis pela assistência farmacêutica no processo de planejamento em saúde em municípios brasileiros. Método: Para este estudo foram utilizados dados da pesquisa "A assistência farmacêutica nas Redes de Atenção à Saúde: um recorte nas regiões do Projeto QualiSUS-Rede", cuja coleta ocorreu de dezembro de 2013 a julho de 2015 em 485 municípios e Distrito Federal, onde foram entrevistados os responsáveis pela assistência farmacêutica nos municípios. Tal estudo teve delineamento transversal, e as variáveis analisadas para o recorte aqui tratado foram as relativas ao perfil dos profissionais responsáveis pela assistência farmacêutica e a participação destes na elaboração e conhecimento dos conteúdos relacionados à assistência farmacêutica nos instrumentos de planejamento orçamentário (PPA, LOA e LDO) e da saúde (PS, PAS, RAG). Verificou-se também a inserção dos instrumentos de planejamento relativos ao período de estudo no Sistema SARGSUS. Resultados: Observou-se uma baixa participação dos responsáveis $(n=417)$ na elaboração dos instrumentos de planejamento orçamentário e da saúde, com maior participação na elaboração do PPA (24,0\%) e do PS (52,0\%). Em relação à PAS e ao RAG, observou-se maior conhecimento da presença de ações relacionadas à assistência farmacêutica por aqueles que participaram da sua elaboração (67,0\% e $68,8 \%$ respectivamente). Conclusão: Os resultados apontam baixa participação dos atores responsáveis pela assistência farmacêutica municipal na elaboração das ferramentas de planejamento dos municípios e pouco conhecimento das ações previstas, e sugerem que os instrumentos de planejamento produzidos podem não condizer com a realidade dos serviços e com a alocação racional dos recursos aplicáveis à assistência farmacêutica.
\end{abstract}

Palavras-chave: planejamento em saúde; assistência farmacêutica; planejamento participativo.

'Programa de Pós-graduação em Saúde Coletiva, Faculdade de Ciências da Saúde, Universidade de Brasília (UnB) - Brasília (DF), Brasil.

2Faculdade de Ciências Médicas, Universidade de Campinas (UNICAMP) - Campinas (SP), Brasil.

${ }^{3}$ Instituto de Pesquisa e Apoio ao Desenvolvimento Social (IPADS) - Campinas (SP), Brasil.

${ }^{4}$ Departamento de Farmácia, Faculdade de Ciências da Saúde, Universidade de Brasília (UnB) - Brasília (DF), Brasil.

Trabalho realizado na Universidade de Brasília (UnB) - Brasília (DF), Brasil.

Endereço para correspondência: Letisa Comparin Dalla Nora - Programa de Pós-graduação em Saúde Coletiva, Faculdade de Ciências da Saúde, Universidade de Brasília (UnB), Campus Universitário Darcy Ribeiro - CEP: 70910-900 - Brasília (DF), Brasil - Email: letisadalla1@gmail.com

Fonte de financiamento: 0 Projeto QualiSUS-Rede foi financiado pelo Ministério da Saúde através do IBRD - Banco Internacional para Reconstrução e

Desenvolvimento (Banco Mundial).

Conflito de interesses: nada a declarar. 


\begin{abstract}
Background: The development of Pharmaceutical Assistance, as well as all areas of action of the SUS, is the responsibility of the three managing instances (municipal, state and federal). In this context, the participation of the actors responsible for its management in the forecast planning budget resources for allocation in this area is fundamental, according to their capacity and priority, taking into account the scarcity of resources and the needs verified. Objective: To describe the participation of the ones involved in the pharmaceutical assistance, regarding instruments development for budgeting and health planning for their municipalities. Method: This paper has analyzed information produced by the project "A assistência farmacêutica nas Redes de Atenção à Saúde: um recorte nas regiões do Projeto QualiSUS-Rede", where data gathering, conducted from December 2013 to July 2015 , in 485 municipalities and the Federal District, was handled through the interview of those in charge of the Pharmaceutical Assistance in the municipalities. The study was cross-sectional and analyzed variables related to the profile of the professionals responsible for Pharmaceutical Assistance regarding professional training, workload and work relationship, as well as the participation of these professionals in the elaboration of the budget planning (PPA, LOA, LDO) and health instruments (PS, PAS, RAG). Complimentarily, we verified the insertion of the planning instruments related to the study period in the SARGSUS System. Results: Pharmaceutical assistance $(n=417)$ was low as in the elaboration of the budget planning and health instruments, with a greater participation in the elaboration of PPP (24.0\%) and PS (52.0\%). Regarding PAS and RAG, there was a greater knowledge of the presence of actions related to pharmaceutical assistance by those who participated in its elaboration $(67.0 \%$ and $68.8 \%$, respectively). Conclusion: The data depict unsatisfactory engagement of the actors responsible for Pharmaceutical Assistance in elaboration and awareness of the actions foreseen at the municipalities' planning tools and suggests the produced planning instruments may not be linked with the real situation of the health services as well as with the rational use of financing for the Pharmaceutical Assistance.
\end{abstract}

Keywords: health planning; pharmaceutical services; participative planning.

\section{INTRODUÇÃO}

No campo da saúde pública brasileira, a instituição de políticas planificadas foi tardia. As políticas públicas caracterizavam-se pelo caráter autoritário e por realizarem-se por meio de campanhas sanitárias e programas especiais do Ministério da Saúde, de forma verticalizada ${ }^{1}$.

Nesse sentido, o Movimento pela Reforma Sanitária e a incorporação dos princípios e diretrizes instituídos na Constituição Federal de 1988 favoreceram o desenvolvimento do planejamento em saúde, em especial pela descentralização, com direção única em cada esfera de governo ${ }^{2}$.

No âmbito da assistência farmacêutica, em 1998 foi instituída a Política Nacional de Medicamentos (PNM), com o objetivo de garantir a necessária segurança, eficácia e qualidade dos medicamentos, a promoção do uso racional e o acesso da população àqueles considerados essenciais à reorientação do modelo de assistência farmacêutica fundamentada na descentralização das ações, contemplando a adoção de uma Relação Nacional de Medicamentos Essenciais, o planejamento adequado e oportuno e a redefinição das atribuições das três instâncias de gestão ${ }^{3}$. Em 2004, foi aprovada a Política Nacional de Assistência Farmacêutica (PNAF), como uma política norteadora para a formulação de políticas setoriais e definida como parte integrante da Política Nacional de Saúde, envolvendo um conjunto de ações voltadas à promoção, proteção e recuperação da saúde e garantindo os princípios da universalidade, integralidade e equidade ${ }^{4}$.

Com a descentralização dos recursos financeiros federais para a aquisição de medicamentos que ocorreu em 2005, foi instituída legalmente a necessidade de planejamento nesta área ${ }^{5}$. Levando-se em consideração que a maioria das intervenções em saúde envolve o uso de medicamentos, é imprescindível que a assistência farmacêutica seja vista sob ótica integral, e que as etapas que a constituem estejam bem estruturadas e articuladas para garantir de fato a atenção integral à saúde 5 .

Como o desenvolvimento da assistência farmacêutica, assim como de todas as áreas de atuação do SUS, é de responsabilidade das três instâncias gestoras (municipal, estadual e federal) o compromisso com sua organização e estruturação, para além da oferta de medicamentos à população. Nesse contexto, é fundamental a participação dos atores responsáveis pela assistência farmacêutica no planejamento da previsão de recursos orçamentários próprio para alocação nessa área, conforme sua capacidade e prioridade, levando-se em conta a escassez de recursos e as necessidades verificadas ${ }^{5}$.

Para Tancredi et al. ${ }^{6}$, o planejamento parte do pressuposto de que os atores que planejam possuem a capacidade de conduzir processos de mudança, por serem detentores de um conjunto de conhecimentos teóricos e práticos inerentes a sua qualificação técnica e experiência. Esse processo permite a esses sujeitos programarem-se para tornar possível o alcance das metas estabelecidas e a interação com a realidade, tanto no plano econômico quanto no plano social ${ }^{6}$.

Sendo assim, este trabalho tem como objetivo descrever a participação dos profissionais responsáveis pela assistência farmacêutica nos processos de planejamento em saúde e o seu conhecimento acerca das ações da assistência farmacêutica constantes nos instrumentos de planejamento orçamentário e da saúde elaborados no âmbito municipal.

\section{MÉTODO}

Trata-se de uma análise cuja fonte de dados provém da pesquisa 'Assistência Farmacêutica nas Redes de Atenção à Saúde: um recorte nas regiões do QualiSUS-Rede', a qual teve como 
objetivo a identificação da situação dos serviços farmacêuticos, técnicos gerenciais e assistenciais nas 15 Regiões prioritárias do QualiSUS-Rede 7 .

O estudo teve delineamento transversal, com coleta de dados entre o período de dezembro de 2013 a julho de 2015 em 485 municípios e Distrito Federal, de 43 regiões de saúde ${ }^{8}$, distribuídos nas cinco regiões do país e que faziam parte das Regiões QualiSUS-Rede em setembro de 2013.

Para esta análise foram utilizados os dados do instrumento estruturado que foi aplicado aos responsáveis pela assistência farmacêutica dos municípios investigados. As variáveis analisadas foram as relativas ao perfil dos profissionais responsáveis pela assistência farmacêutica quanto à formação profissional, carga horária e vinculo de trabalho, bem como a participação desses profissionais na elaboração dos instrumentos de planejamento orçamentário, o Plano Plurianual (PPA), a Lei de Diretrizes Orçamentárias (LDO) e a Lei Orçamentária Anual (LOA), e dos instrumentos de planejamento da saúde, o Plano de Saúde (PS), incluindo-se aqui os Planos Municipais de Saúde e o Plano Distrital de Saúde, a Programação Anual de Saúde (PAS) e o Relatório Anual de Gestão (RAG). Além disso, foi investigado também o conhecimento desses atores a respeito do conteúdo relacionado à assistência farmacêutica na LOA, na PAS e no RAG.

De forma complementar, verificou-se a inserção do Plano de Saúde 2014-2017, das Programações Anuais de Saúde 2013, 2014 e 2015 e dos Relatórios Anuais de Gestão 2013, 2014 no Sistema de Apoio ao Relatório de Gestão (Sistema SARGSUS) para todos os municípios investigados neste trabalho, por meio de consulta ao sítio eletrônico ${ }^{9}$, mais especificamente do Consolidado da Situação dos Instrumentos de Planejamento. O documento Consolidado utilizado foi o último publicado até a conclusão deste trabalho, que data de 09 de dezembro de 2015. As informações sobre as Programações Anuais de Saúde 2013 e 2014, que não constam no Consolidado, foram consultadas nos Relatórios Gerenciais da Situação dos Instrumentos de Planejamento, no período de dezembro de 2015 a março de 2016.

Considerando o período em que as entrevistas aplicadas aos responsáveis pela assistência farmacêutica foram realizadas, e que as questões se referem a fatos do passado, a investigação entre a participação na elaboração dos instrumentos com sua postagem no SARGSUS foi realizada para o PS 2014-2017, para o PAS 2014 e para o RAG 2013, já que a elaboração desses instrumentos deveria ocorrer teoricamente no final de 2013 para o PS e o PAS, e até o primeiro trimestre de 2014 para o RAG.

A análise dos resultados foi realizada pela inferência estatística descritiva, onde foram calculadas as frequências absolutas simples e relativas, com suporte do sistema para análises estatísticas do programa Epi Info ${ }^{\mathrm{TM}}$ 7.1.5.
O estudo foi aprovado pelo Comitê Nacional de Ética em Pesquisa (CONEP), Parecer 399.423 de 18 de setembro de 2013. Todas as entrevistas foram precedidas de assinatura de Termo de Consentimento Livre e Esclarecido (TCLE).

\section{RESULTADOS}

Dos 485 municípios investigados no estudo, $14 \%$ não possuíam responsável pela assistência farmacêutica (RAF) formalmente instituído. Sendo assim, foram entrevistados 417 RAFs dos municípios e do Distrito Federal, que configuram o recorte desta análise.

A investigação revelou que, em relação à formação profissional, do total de entrevistados $(n=417)$, a maioria é de farmacêuticos $(90,2 \%)$ e com jornada de trabalho de 40 horas semanais $(30,9 \%)$. Em relação ao vínculo de trabalho, 46,0\% declararam ser concursados, $34,8 \%$ contratados e, dentre os entrevistados, $11,5 \%$ possuem especialização na área de assistência farmacêutica, e 2,4\% cursaram mestrado na área. Ressalta-se ainda que à frente da assistência farmacêutica dos municípios estão enfermeiros $(1,2 \%)$, técnicos de enfermagem $(0,9 \%)$, odontólogo $(0,2 \%)$, veterinário $(0,2 \%)$ e assistente social $(0,2 \%)$, dentre outras formações. Verificou-se que a maioria dos municípios possui apenas um farmacêutico, sendo que em 07 municípios não havia nenhum farmacêutico no quadro de colaboradores.

Em relação à participação dos RAF na elaboração da programação orçamentária (PPA, LDO e LOA), a maioria dos profissionais entrevistados informou não ter participado da elaboração da LOA e LDO, sendo observada uma maior frequência de participação na elaboração do PPA $(24,0 \%)$ (Figura 1).

Quando questionados sobre o conhecimento da LOA vigente, a maioria $(70,1 \%)$ respondeu negativamente. Quanto ao detalhamento dos conteúdos apresentados na LOA, a Tabela 1 apresenta o comparativo entre a participação dos profissionais em sua elaboração e seu conhecimento sobre o conteúdo deste documento.

Verifica-se que uma maior proporção de entrevistados que afirmou ter participado da elaboração do instrumento confirmou a previsão de recursos para as ações de aquisição e estruturação na área de assistência farmacêutica na LOA. Em relação à previsão de ações para além da aquisição e estruturação, a maior parte dos entrevistados que disseram participar da preparação da LOA informaram que não havia recursos para este quesito, que inclui ações em educação, por exemplo.

Em relação à participação na elaboração dos instrumentos de planejamento da saúde (PS, PAS e RAG), pode-se observar que, em relação ao os, menos da metade dos responsáveis pela assistência farmacêutica informaram ter participado (52,0\%). E da elaboração da PAS, apenas 33,2\% referiram participar. Em relação ao RAG, grande parte, $60,0 \%$, relatou não ter participado da elaboração deste documento (Figura 2). 


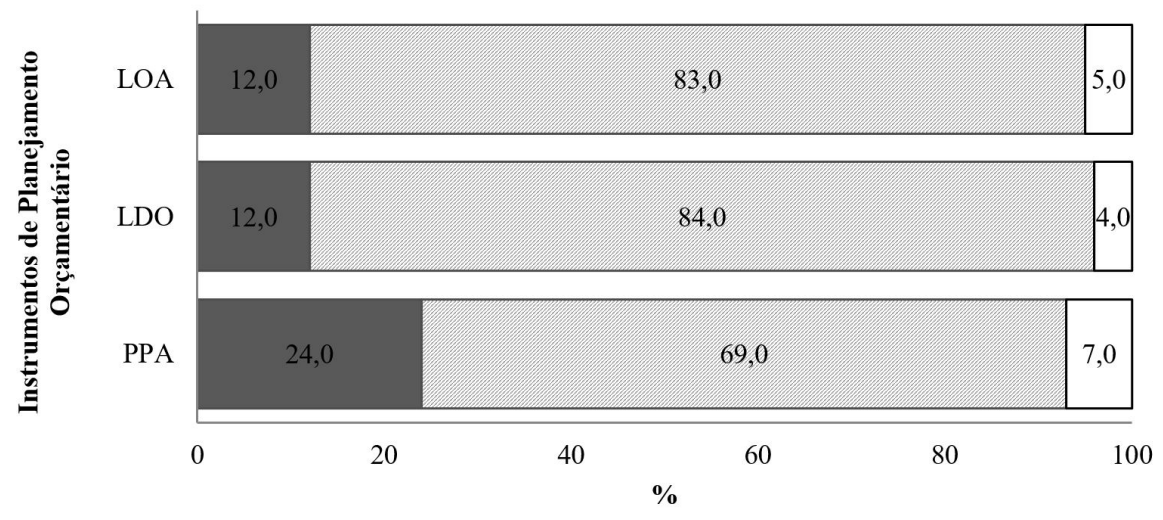

• Sim $\square$ Não ロNão sei

Figura 1. Participação do responsável pela assistência farmacêutica (RAF) na elaboração do Plano Plurianual (PPA), da Lei de Diretrizes Orçamentárias (LDO) e da Lei Orçamentária Anual (LOA). Regiões QualiSUS Rede, 2015.

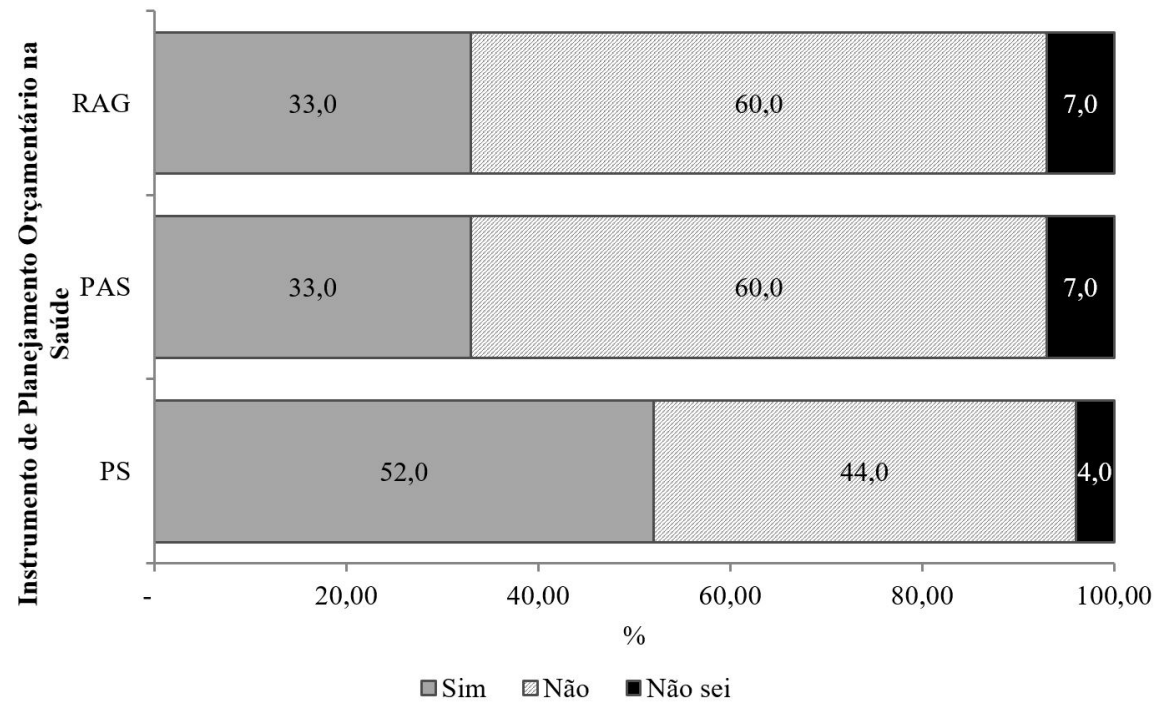

Figura 2. Participação do responsável pela assistência farmacêutica (RAF) na elaboração do Plano de Saúde (PS), da Programação Anual de Saúde (PAS) e do Relatório Anual de Gestão (RAG). Regiões QualiSUS Rede, 2015.

Tabela 1. Conhecimento do responsável pela assistência farmacêutica (RAF) sobre o conteúdo inerente à assistência farmacêutica na Lei Orçamentária Anual (LOA) de seu município, segundo sua participação na elaboração ( $n=412)$. Regiões QualiSUS Rede, 2015.

\begin{tabular}{ccc} 
Conhecimento do RAF sobre a & \multicolumn{2}{c}{$\begin{array}{c}\text { Participação na } \\
\text { elaboração da LOA }\end{array}$} \\
\cline { 2 - 2 } presença na LOA & Sim & Não \\
\cline { 2 - 2 } & $\mathbf{n}(\%)$ &
\end{tabular}

Ações para aquisição

\begin{tabular}{llc} 
Sim & $44(88,0)$ & $128(35,3)$ \\
Não & $06(12,0)$ & $235(64,7)$ \\
Total & $\mathbf{5 0 ( 1 0 0 , 0 )}$ & $\mathbf{3 6 3}(\mathbf{1 0 0 , 0})$ \\
Ações para estruturação & & \\
Sim & $28(56,0)$ & $57(15,7)$ \\
Não & $22(44,0)$ & $305(84,3)$ \\
Total & $\mathbf{5 0 ( 1 0 0 , 0 )}$ & $\mathbf{3 6 2 ( 1 0 0 , 0 )}$ \\
Ações para além da aquisição e & & \\
estruturação & & \\
Sim & $15(30,0)$ & $19(5,2)$ \\
Não & $35(70,0)$ & $343(94,8)$ \\
Total & $\mathbf{5 0 ( 1 0 0 , 0 )}$ & $\mathbf{3 6 2}(\mathbf{1 0 0 , 0 )}$ \\
\hline
\end{tabular}

Referente à Programação Anual da Saúde, foram discriminadas pelos entrevistados algumas ações da assistência farmacêutica previstas neste instrumento. Dentre elas, as ações relacionadas à informatização foram as mais apontadas (22,5\%), seguida da previsão de capacitações/educação permanente $(21,8 \%)$, reformas (17,7\%), ampliação de recursos humanos (13,2\%), e em último lugar ficou a previsão de ações de construção nas unidades (10,8\%).

Em relação à PAS e ao RAG, observou-se maior conhecimento da presença de ações relacionadas à assistência farmacêutica no instrumento por aqueles que participaram da sua elaboração (67,0\% e 68,8\% respectivamente) (Tabela 2$)$.

De forma complementar, foi verificada a relação entre a postagem dos Planos Municipais de Saúde 2014-2017, das Programações Anuais de Saúde 2013, 2014 e 2015 e dos Relatórios Anuais de Gestão 2013 e 2014 dos 417 municípios investigados no Sistema SARGSUS. Seguem os resultados na Tabela 3. 
Tabela 2. Conhecimento do responsável pela assistência farmacêutica (RAF) sobre o conteúdo inerente à assistência farmacêutica na Programação Anual de Saúde (PAS) e No Relatório Anual de Gestão (RAG) de seu município, segundo sua participação na elaboração. Regiões QualiSUS Rede, 2015.

\begin{tabular}{ccc} 
& \multicolumn{2}{c}{$\begin{array}{c}\text { Participação na elaboração } \\
\text { da PAS }\end{array}$} \\
\cline { 2 - 3 } $\begin{array}{c}\text { Conhecimento do RAF sobre a } \\
\text { presença na PAS e no RAG }\end{array}$ & Sim & Não \\
\cline { 2 - 3 } & $\mathbf{n}(\%)$
\end{tabular}

Ações da AF descritas na PAS $(\mathrm{n}=376)$

\begin{tabular}{lcc} 
Sim & $120(67,0)$ & $17(7,4)$ \\
Não & $59(33,0)$ & $214(92,6)$ \\
Total & $\mathbf{1 7 9 ( 1 0 0 , 0 )}$ & $\mathbf{1 9 7}(\mathbf{1 0 0 , 0})$ \\
\multicolumn{3}{c}{ Participação na elaboração do } \\
RAG \\
Ações da AF descritas no \\
último RAG (n=415) \\
Sim \\
Não & $107(77,5)$ & $42(15,2)$ \\
Total & $31(22,5)$ & $235(84,8)$ \\
Recursos previstos e executados & $\mathbf{1 3 8 ( 1 0 0 , 0 )}$ & $\mathbf{2 7 7}(\mathbf{1 0 0 , 0})$ \\
no último RAG (n=415) & & \\
Sim & $95(68,8)$ & $53(19,3)$ \\
Não & $43(31,2)$ & $224(81,7)$ \\
Total & $\mathbf{1 3 8 ( 1 0 0 , 0 )}$ & $\mathbf{2 7 7}(\mathbf{1 0 0 , 0})$ \\
\hline
\end{tabular}

Tabela 3. Participação dos responsáveis pela assistência farmacêutica no planejamento da saúde e a inserção dos documentos no Sistema de Apoio ao Relatório de Gestão (Sistema SARGSUS), Regiões QualiSUS Rede, 2015.

\begin{tabular}{lcc}
\multirow{2}{*}{ Situação no SARGSUS } & \multicolumn{2}{c}{ Participação na elaboração } \\
\cline { 2 - 3 } PS 2014-2017 (n=415) & Sim n (\%) & Não n (\%) \\
Sim & $40(85,1)$ & $319(86,7)$ \\
Não & $5(10,6)$ & $23(6,2)$ \\
Não atualizou & $2(4,3)$ & $26(7,1)$ \\
Total & $\mathbf{4 7}(\mathbf{1 0 0 , 0})$ & $\mathbf{3 6 8}(\mathbf{1 0 0 , 0 )}$ \\
PAS 2014 (n=389) & & \\
Sim & $92(73,0)$ & $150(57,1)$ \\
Não & $9(7,2)$ & $18(6,8)$ \\
Não atualizou & $25(19,8)$ & $95(36,1)$ \\
Total & $\mathbf{1 2 6}(\mathbf{1 0 0 , 0})$ & $\mathbf{2 6 3}(\mathbf{1 0 0 , 0 )}$ \\
RAG 2013 (n=414) & & \\
Sim & $128(95,5)$ & $261(93,2)$ \\
Não & $06(4,5)$ & $19(6,8)$ \\
Total & $\mathbf{1 3 4}(\mathbf{1 0 0 , 0})$ & $\mathbf{2 8 0}(\mathbf{1 0 0 , 0 )}$ \\
\hline
\end{tabular}

Fonte: Sistema de Apoio ao Relatório de Gestão ${ }^{9}$

Resultados conflitantes foram encontrados ao analisar a presença dos Relatórios Anuais de Gestão e respectivas Programações Anuais de Saúde. Entre os 417 Municípios e o Distrito Federal que participaram deste estudo, foram encontrados 225 municípios que inseriram o RAG 2013 no SARGSUS, entretanto, alegaram não ter Programação Anual de Saúde ou não postaram informações a respeito deste instrumento no Sistema. Para o ano de 2014, este mesmo caso ocorreu com 107 municípios. Ao analisar a presença do Plano de Saúde 2014-2017 com o Relatório Anual de Gestão 2014 (o RAG 2015 não está disponível no período da consulta), verificou-se um total de 22 municípios que postaram o RAG, porém não apresentam o PS.

\section{DISCUSSÃO}

Os resultados apontaram para a baixa participação dos responsáveis pela assistência farmacêutica na elaboração das ferramentas de planejamento orçamentário e da saúde de seus municípios. Em relação ao conhecimento da presença de ações referentes à assistência farmacêutica em algumas ferramentas de planejamento como a LOA, a PPA e o RAG, observou-se maior conhecimento por parte daqueles que participaram da elaboração dos instrumentos.

A participação ou não dos profissionais na elaboração das ferramentas do planejamento apresenta diferentes graus de implicação. Em relação ao PPA, que estabelece as diretrizes, objetivos e metas da Administração Pública, a falta de participação dos profissionais relacionados à assistência farmacêutica implicará ausência de pauta para as questões da área por um período de quatro anos. Sendo este a mais abarcante ferramenta do planejamento público, pois integra as ações planejadas para médio prazo e os meios orçamentários para viabilização do aporte de recursos, esta perda tem impacto direto pelo período de sua vigência ${ }^{10}$.

Considerando a complexidade das pautas relacionadas ao abastecimento de medicamentos e à qualificação dos serviços, é de fundamental importância que as questões relacionadas à assistência farmacêutica estejam devidamente contempladas no PPA, da forma abrangente e complexa que o setor exige, e para tanto ressalta-se a importância da participação dos profissionais que conhecem este campo.

Ainda em relação ao planejamento orçamentário, a LDO orienta quanto à formulação da Lei Orçamentária Anual (LOA) do ano correspondente, por meio do estabelecimento de diretrizes, objetivos e metas, e é elaborada de acordo com o PPA. Tal instrumento possibilita ajustar as ações de governo às reais possibilidades financeiras do exercício, momento em que são indicados os rumos a serem priorizados neste período, além das previsões de realização de receitas e do controle das despesas públicas. Considerando que a lei orçamentária anual não pode conter dispositivo alheio à previsão da receita e à fixação da despesa, não estando contempladas na LDO as questões da assistência farmacêutica, consequentemente não haverá previsão orçamentária para elas na LOA, o que implica que, mesmo sendo estas questões estruturantes para a saúde pública municipal, não poderão receber o adequado apoio financeiro para sua realização ${ }^{11}$.

A questão da alocação dos recursos no orçamento deve ser mais bem verificada em decorrência da cultura estratégica dos governos de tentar manter os recursos num plano mais 
agregado da classificação orçamentária, de forma a facilitar a remanejamento entre programas e ações durante sua gestão ${ }^{12}$. Esse comportamento é desfavorável à adequada avaliação do planejamento realizado e pode revelar falta de previsibilidade de cenários.

Em se tratando do planejamento na saúde, mais especificamente em relação à elaboração do Plano de Saúde, a maioria dos entrevistados informou não ter participado. Este instrumento promove visibilidade sobre as responsabilidades dos gestores, e sua elaboração é considerada estratégica para a organização do setor saúde. Como o PPA, as ações previstas no PS têm duração de quatro anos e são desdobradas na Programação Anual de Saúde. Desta, pouco mais de um terço dos RAF disseram participar, o que demonstra que a falta de planejamento participativo se agrava inversamente à necessidade de que sua elaboração deva ser feita por quem conhece no detalhe a realidade do setor, considerando a pontualidade de ações e programas que fazem parte do planejamento anual da saúde.

Na planificação estratégica situacional, Matus ${ }^{13}$ aborda como uma das etapas do planejamento a elaboração de cenários de cálculo, que são prognósticos sobre o desenrolar de situações externas. Segundo Schwartz ${ }^{14}$, os cenários são uma ferramenta que permite percepções sobre ambientes futuros alternativos e o planejamento por cenários possibilita ao administrador estar preparado para eles.

Para enfrentamento das mudanças de cenário, bem como para desenhar as estratégias que configuram o planejamento, são necessárias várias visões que permitam a compreensão das situações problema definidos previamente pelos envolvidos como prioritárias. Nesse sentido, a programação anual de saúde carece da prospecção dos atores sociais envolvidos na ação.

Resultados semelhantes foram encontrados em outro estudo, realizado com municípios do Estado de Santa Catarina. Dos seis municípios pesquisados, apenas um informou conhecimento por parte de todos os farmacêuticos entrevistados sobre o Plano de Saúde ${ }^{15}$. A baixa participação dos profissionais na elaboração do Plano de Saúde e da Programação Anual de Saúde pode acarretar em prejuízos ao alcance dos objetivos propostos. Tendo em vista os resultados apresentados, pode-se supor que estes profissionais podem desconhecer as prioridades para seu setor, ou ainda discordar das definições de prioridades realizadas e não aderir a elas, uma vez que não partilharam das etapas de diagnóstico das situações-problema e da elaboração do conteúdo propositivo para o período. A não adesão ou sensibilização dos atores em questão pode acarretar em problemas para o serviço, uma vez que são estes os profissionais que devem trabalhar para o alcance dos objetivos e metas estabelecidos.

A "gestão participativa", uma obrigação legal dos gestores no âmbito do SUS, é um processo que advém do compromisso assumido por todos os participantes, o que confere legitimidade ao
Plano e facilita a implantação de mecanismos de responsabilização. No âmbito da responsabilização, deve-se considerar ainda o desafio da compreensão da organização por parte do profissional e dos valores adotados pela instituição em que está inserido como forma de comprometimento. Sendo assim, dadas as características das organizações de saúde e da falta de participação dos entrevistados na elaboração do planejamento da saúde, justifica-se a admissão de um conjunto de práticas que apresentem a cultura da gestão por compromissos, em vez de um sistema que apresente puramente a gestão estratégica ${ }^{16}$.

Ficou claro no presente trabalho o desconhecimento dos RAFs sobre a previsão orçamentária para aquisição de medicamentos e para estruturação. Pela sua natureza técnica, o planejamento orçamentário em geral é elaborado por especialistas da área financeira, muitas vezes sem a suficiente participação dos especialistas da área da saúde durante a fase do diagnóstico do planejamento $^{12}$. Desta forma, entende-se que a qualificação e o empoderamento dos profissionais da área afim poderiam favorecer sua participação e a melhoria dos processos de elaboração do planejamento orçamentário municipal e do Distrito Federal.

Referente ao Relatório Anual de Gestão, a maioria dos entrevistados afirmou não ter participado de sua elaboração e não saber se as ações da assistência farmacêutica estavam contempladas neste. Considerando o processo 'aprendizagemcorreção-aprendizagem' de Matus ${ }^{13}$, nada mais lógico que os profissionais que executam as atividades façam também a avaliação de seu desempenho. Entretanto, da mesma forma que não participaram da elaboração dos instrumentos de planejamento, estão também afastados das atividades de prestação de contas. Se a planificação for entendida como um processo cíclico de correção de rumos, de forma que a cada mudança de cenário seja corrigida a trajetória para alcançar o objetivo almejado, faz-se necessário que os atores apontem as variações não favoráveis ao andamento orquestrado dos processos.

A participação dos profissionais da assistência farmacêutica no planejamento e na prestação de contas produz conhecimento e qualifica estes atores. Ao realizar uma visão futura na tentativa de solucionar os problemas da organização, identificados de forma participativa, há a incorporação do entendimento estratégico pela equipe, que pode ser aplicado em decisões futuras ${ }^{17}$. Entende-se que estes ganhos não serão sentidos pela maioria das equipes de saúde dos municípios estudados, já que os profissionais que estão à frente da assistência farmacêutica ficam em grande parte à margem dos processos de diagnóstico das situações-problema de sua área e das instâncias de decisão sobre os rumos a serem seguidos.

Quando analisados os dados referentes à inserção dos documentos do planejamento da saúde no SARGSUS, para a maioria dos municípios que inseriram os Planos de Saúde no sistema, os entrevistados informaram não terem participação em 
sua elaboração. O mesmo foi observado no caso da Programação Anual de Saúde.

Referente aos Relatórios Anuais de Gestão, a despeito da não participação dos entrevistados em sua elaboração, a maioria dos RAG 2013 e 2014 foi inserido no SARGSUS. Infere-se com isto que o cumprimento legal da prestação de contas é priorizado nos municípios estudados, uma vez que nem mesmo os Planos Municipais de Saúde foram mais postados no sistema eletrônico. Destaca-se que, para vários municípios que não informaram as Programações Anuais de Saúde 2013 e 2014 no SARGSUS, haviam os Relatórios Anuais de Gestão dos respectivos anos. Isto denota fragilidade e desarticulação na prestação de contas do cumprimento das metas anuais, sem previsão das prioridades no instrumento do planejamento anual em saúde.

O Relatório de Gestão é uma ferramenta fundamental para a realização do processo de avaliação das ações e formulação de um novo planejamento. A realidade que permeia sua formulação, entretanto, é de insuficiência de recursos humanos qualificados para apoiar seu processo de desenvolvimento e de baixo conhecimento dos gestores sobre planejamento, programação e avaliação, sobremaneira na questão conceitual ${ }^{17}$. Com base nos achados deste estudo, em que o RAG pode estar sendo realizado sem a prévia elaboração da Programação Anual de Saúde, é questionável a eficiência deste instrumento no alcance dos objetivos a que se propõe, como o de ser um mecanismo estratégico para o aperfeiçoamento da gestão, com a avaliação dos resultados e a reorganização das práticas de saúde às necessidades da população.

A realidade apontada por alguns estudos em relação à assistência farmacêutica no país reflete a falta de planejamento na oferta de ações e serviços nos municípios brasileiros. Grande parte dos municípios brasileiros perece ainda com baixa disponibilidade e descontinuidade na oferta de medicamentos essenciais nas Unidades Básicas de Saúde, além de precárias condições de armazenamento dos produtos, bem como listas municipais de medicamentos que não refletem as necessidades locais, ocasionando falta de acesso aos medicamentos pela população ${ }^{18,19}$. A área da assistência farmacêutica, relacionada à garantia do abastecimento e ao acesso aos medicamentos, é marcada por atividades previsíveis, calculadas por normas e diretrizes predeterminadas, como o financiamento, o processo de programação, aquisição e guarda dos produtos.

Cabe destacar que, além das atividades gerenciais, são inerentes à assistência farmacêutica as atividades relacionadas ao uso dos medicamentos, as quais envolvem conhecimentos e habilidades por parte dos farmacêuticos relacionadas à boa comunicação com os usuários de medicamentos e com os outros profissionais envolvidos em seu tratamento ${ }^{20}$. Neste quesito também podemos verificar carências, uma vez que vários problemas de saúde estão relacionados ao uso de medicamentos, e são causas importantes de procura de atendimentos de urgência e emergência no Brasil ${ }^{21}$. Citam-se, ademais, as dificuldades vinculadas à não adesão à farmacoterapia, que representa um verdadeiro desafio para o sistema de saúde, com escassez de serviços dedicados ao fortalecimento da adesão dos usuários aos tratamentos propostos. O Ministério da Saúde reconhece esta fragilidade e já apontou como estratégico o desenvolvimento de metodologias para fortalecer a adesão ao tratamento das doenças crônicas ${ }^{22}$.

Tomando-se a existência de dois grupos de funções gerenciais, a desenvolvida nas unidades de execução dos serviços de saúde e a realizada nas instâncias de comando e coordenação, Ferreira, 2004, destaca que, para a qualificação constante dos serviços prestados à comunidade, o gestor deve tomar a negociação como ferramenta na condução do processo de trabalho. Cabe destacar, por sua vez, que compete a esses profissionais qualificarem-se adequadamente para essas atividades, o que não afasta a importância do incentivo institucional para que essas capacitações aconteçam ${ }^{23}$. A maioria dos entrevistados informou não ter formação na área de gestão, incluídos aí cursos de especialização, mestrado e doutorado.

Este cenário torna-se mais fragilizado com o dado deste estudo sobre a previsão de recursos orçamentários específicos para o desenvolvimento das ações da assistência farmacêutica, para além do processo de aquisição e de estruturação das unidades de atendimento e de armazenamento de medicamentos e insumos. Dentre estas ações, podemos citar as relacionadas à educação permanente para os colaboradores, em que a grande maioria dos participantes informou que não havia previsão orçamentária para essas ações. Há que se investigar, ainda, se a pouca qualificação ocorre por falta de interesse dos farmacêuticos pelo planejamento, bem como pelo não entendimento de seu papel nas organizações de saúde.

Para uma gestão mais assertiva dos sistemas de saúde, é necessária uma melhor compreensão da estrutura, da organização e dos instrumentos de planejamento desses sistemas. O desconhecimento dessas bases pode gerar uma prática profissional descontextualizada, “[...] exercitada a partir do senso comum ou do voluntarismo político" (p. 15), o que muitas vezes costuma ser a regra ${ }^{24}$. Dessa forma, não é incomum o planejamento orçamentário estar a cargo exclusivamente das áreas administrativa e financeira, que muitas vezes não convidam os gestores da saúde para partilharem desse processo ${ }^{12}$. Destaca-se também que o conhecimento das despesas, da projeção das receitas, dos riscos envolvidos nos projetos permite o controle das atividades financeiras do setor, sendo imperativo a garantia da transparência visando ao fortalecimento da gestão pública responsável e efetiva.

A visão reducionista em relação aos serviços farmacêuticos, ainda observada, pode ser revertida com seu reconhecimento como uma área de gestão, estratégica, e não apenas como um ponto de dispensação de medicamentos ${ }^{25}$. Sua gestão, em parte, 
está ligada à capacidade de alocar e gerenciar recursos técnicos e administrativos para garantir o acesso e o uso racional de medicamentos ${ }^{26}$. Deficiências nesta capacidade comprometem o alcance deste objetivo, como a baixa qualificação dos responsáveis por estas ações, ou a não institucionalização da assistência farmacêutica na estrutura organizacional como uma instância autônoma ${ }^{26}$.

A julgar pela não participação dos profissionais no planejamento estratégico de atividades de sua responsabilidade, inerentes ao seu cotidiano, e que as ferramentas de prestação de contas, como o Relatório Anual de Gestão, estão mais presentes no SARGSUS que as de programação, podemos inferir que o planejamento da assistência farmacêutica parece ser elaborado na maioria das vezes de forma cartorial, para atender a requisitos legais.

A falta de uma análise situacional instruída na realidade municipal e do cumprimento de ações ordenadas para um adequado planejamento da assistência farmacêutica pode incorrer em atuações pautadas por demandas emergenciais, caracterizadas pelo improviso. Nesta realidade não há adequado abastecimento da rede de saúde, nem adequado atendimento aos usuários, tampouco promoção do uso racional dos medicamentos.
O estudo realizado apresentou limitações em relação à vinculação dos resultados, pois se trata de respostas autorreferidas, e não de observação direta dos instrumentos de planejamento pesquisados. Entretanto, os dados são importantes do ponto de vista do conhecimento dos atores acerca do assunto, e de sua interação com este tema da gestão pública, fato que confere relevância e pertinência aos resultados obtidos. Ressalta-se também a falta de estudos com o mesmo objetivo e temática deste, de forma que o caráter exploratório ficou a cargo da revisão das teorias relacionadas à matéria em questão.

Atividades de gestão sem planejamento incorrem geralmente em rotinas pautadas por ações emergenciais, da mesma forma que planejamento desvinculado da gestão, realizado sem a participação dos atores envolvidos com o processo, resulta em ferramentas inaplicáveis, não condizentes com a realidade local e que não são capazes de alocar recursos de forma racional.

Os resultados revelam lacunas importantes no planejamento da assistência farmacêutica municipal, que apontam a necessidade de maior participação dos atores na elaboração e conhecimento das ações previstas nas ferramentas de planejamento, com vistas ao atendimento integral dos usuários do SUS.

\section{REFERÊNCIAS}

1. Paim JS. Saúde, política e reforma sanitária. Salvador: UFBA; 2002.

2. Paim JS. Reforma sanitária brasileira: contribuição para a compreensão e crítica. Salvador: Edufba; 2008.

3. Brasil. Portaria GM/MS n 3.916, de 30 de outubro de 1998. Aprova a Política Nacional de Medicamentos. Diário Oficial da União [Internet], Brasília, 10 de novembro de 1998 [citado em 2015 Out 6]. Disponível em: http://bvsms. saude.gov.br/bvs/saudelegis/gm/1998/prt3916_30_10_1998.html

4. Brasil. Ministério da Saúde. Conselho Nacional de Saúde. Resolução no 338, de 6 de maio de 2004. Aprova a Política Nacional de Assistência Farmacêutica e estabelece seus princípios gerais e eixos estratégicos. Diário Oficial da União [Internet], Brasília, 20 de maio de 2004 [citado em 2014 Nov 17]. Disponível em: http://bvsms.saude.gov.br/bvs/saudelegis/cns/2004/ res0338_06_05_2004.html

5. Brasil. Ministério da Saúde. Secretaria-Executiva. Subsecretaria de Planejamento e Orçamento. Sistema de planejamento do SUS: instrumentos básicos. Brasília: Ministério da Saúde; 2006. (Série B. Textos Básico de Saúde. Série Cadernos de Planejamento; 2).

6. Tancredi FB, Barrios SRL, Ferreira JHG. Planejamento em saúde. São Paulo: Universidade de São Paulo; 1998. p. 5-13. (Saúde \& Cidadania; 2).

7. Brasil. Ministério da Saúde. Secretaria Executiva. Departamento de Economia da Saúde, Investimento e Desenvolvimento. Unidade de Gestão do Projeto - UGP. QualiSUS-Rede: manual operacional: documento-base [Internet]. Brasília: Ministério da Saúde; 2010. Vol. 1 [citado em 2015 Out 24]. Disponível em: http://portalsaude.saude.gov.br/images/pdf/2014/outubro/08/ Vol1DocBaseQualiSUS-RedeWeb.pdf

8. Brasil. Ministério da Saúde. SAGE: Sala de Apoio à Gestão Estratégica [Internet]. Brasília; 2016 [citado em 2016 Mar 23]. Disponível em: http:// sage.saude.gov.br/
9. Brasil. Ministério da Saúde. SargSUS [Internet]. Brasília; 2016 [citado em 2016 Mar 23]. Disponível em: http://aplicacao.saude.gov.br/sargsus

10. Almeida PR. A experiência brasileira em planejamento econômico: uma síntese histórica [Internet]. 2004 [citado em 2015 Fev 13]. Disponível em: http://www.pralmeida.org/05DocsPRA/ 1277HistorPlanejBrasil.pdf

11. Brasil. Constituição da República Federativa do Brasil de 1988. Diário Oficial da União [Internet], Brasília, 5 de outubro de 1988 [citado em 2016 Fev 29]. Disponível em: http://www.planalto.gov.br/ccivil_03/constituicao/ ConstituicaoCompilado.htm

12. Banco Mundial. Brasil: governança no Sistema Único de Saúde (SUS) do Brasil: melhorando a qualidade do gasto público e gestão de recursos [Internet]. 2007 [citado em 2016 Fev 26]. Disponível em: http://www-wds.worldbank. org/external/default/WDSContentServer/WDSP/IB/2015/08/05/090224b 0828a3c2f/1_0/Rendered/PDF/Brasil000Gover0e0gestao0de0recursos.pdf

13. Matus C. Política, planejamento e governo. Brasília: IPEA; 1993.

14. Schwartz P. A arte da visão de longo prazo: planejando o futuro em um mundo de incertezas. São Paulo: Zumble/Best-Seller; 2000.

15. Leite SN, Farias MR, Manzini F, Mendes DJV, Rover MRM, editores. Gestão da assistência farmacêutica: proposta para avaliação no contexto municipal: a experiência em Santa Catarina 2015. Florianópolis: UFSC; 2015.

16. Rivera FJU. Análise estratégica em saúde e gestão pela escuta. Rio de Janeiro: Fiocruz; 2003. http://dx.doi.org/10.7476/9788575413036.

17. Senge P. A Quinta disciplina: arte e prática da organização que aprende. 2. ed. São Paulo: Círculo do Livro; 1998.

18. Oliveira LC, Assis MM, Barboni AR. Assistência farmacêutica no Sistema Único de Saúde: da política nacional de medicamentos à atenção básica à 
saúde. Cien Saude Colet. 2010;15(Supl 3):3561-7. http://dx.doi.org/10.1590/ S1413-81232010000900031.

19. Helfer AP, Camargo AL, Tavares NU, Kanavos P, Bertoldi AD. Capacidade aquisitiva e disponibilidade de medicamentos para doenças crônicas no setor público. Rev Panam Salud Publica. 2012;31(3):225-32. http://dx.doi. org/10.1590/S1020-49892012000300007. PMid:22569697.

20. Araújo AL, Ueta JM, Freitas OD. Assistência Farmacêutica como um modelo tecnológico em atenção primária à saúde. Rev Cienc Farm Basica Apl. 2009;26(2):87-92.

21. Andreazza RS, Castro MS, Köche PS, Heineck I. Causes of drug-related problems in the emergency room of a hospital in southern Brazil. Gac Sanit. 2011;25(6):501-6. http://dx.doi.org/10.1016/j.gaceta.2011.05.016. PMid:21835509.

22. Brasil. Ministério da Saúde. Secretaria de Vigilância em Saúde. Departamento de Análise de Situação de Saúde. Plano de ações estratégicas para o enfrentamento das doenças crônicas não transmissíveis (DCNT) no Brasil
2011-2022. Brasília: Ministério da Saúde; 2011. (Série B. Textos Básicos de Saúde).

23. Ferreira AS. Competências gerenciais para unidades básicas do Sistema Único de Saúde. Cien Saude Colet. 2004;9(1):69-76. http://dx.doi.org/10.1590/ S1413-81232004000100007.

24. Mendes EV. Os sistemas de serviços de saúde: o que os gestores deveriam saber sobre essas organizações complexas. Fortaleza: Escola de Saúde Pública do Ceará; 2002.

25. Mendes SJ, Manzini F, Farias MR, Leite SN. Gestão da assistência farmacêutica: avaliação de um município Catarinense. Gestão e Saúde. 2014;6(1):4-29.

26. Vieira FS, Zucchi P. Gestão da assistência farmacêutica: análise da situação de alguns municípios. Tempus Actas de Saúde Coletiva. 2015;8(4):11-29. http://dx.doi.org/10.18569/tempus.v8i4.1581.

Recebido em: Mar. 15, 2018 Aprovado em: Jan. 03, 2019 